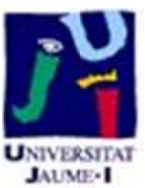

Título artículo / Títol article: Liderazgo transformacional y desempeño grupal: unidos por el engagement grupal

Autores / Autors

Salanova Soria, Marisa ; Martínez Martínez, Isabel M. ; Cruz Ortiz, Valeria

Revista:

Revista de Psicología Social

Versión / Versió:

Postprint de l'autor

Cita bibliográfica / Cita

CRUZ-ORTIZ, Valeria; SALANOVA, Marisa;

bibliogràfica (ISO 690):

MARTÍNEZ, Isabel M. Liderazgo transformacional y desempeño grupal: unidos por el engagement grupal. Revista de Psicología Social, 2013, vol. 28, no 2, p. 183-196

url Repositori UJI:

http://hdl.handle.net/10234/95696 


\title{
Liderazgo transformacional y desempeño grupal: unidos por el engagement grupal
}

\author{
Valeria Cruz-Ortiz, Marisa Salanova e Isabel M. Martínez \\ Universitat Jaume I
}

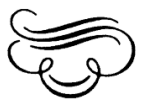

Resumen

Este estudio pone a prueba las relaciones entre liderazgo transformacional, engagement grupal y desempeño grupal, a partir del Modelo de Organizaciones Saludables y Resilientes (HERO - HEalthy E Resilient Organizations) (Salanova, 2008; Salanova, Martínez, Cifre y Llorens, 2009) poniendo de manifiesto el papel mediador del engagement grupal en esta relación. La muestra está compuesta por 519 empleados pertenecientes a 58 equipos de trabajo de 12 Pequeñas y Medianas Empresas (PyMEs) españolas. Los Coeficientes de Correlación Intraclase (CCI1 y CCI2) y el Índice de Desviación Promedio $(A D M(J))$, permiten la agregación de los datos al nivel de equipos de trabajo para el desarrollo de los análisis correspondientes. Los resultados obtenidos a nivel de equipos de trabajo, apoyan la hipótesis planteada, esto es, el engagement grupal media totalmente la relación entre liderazgo transformacional y desempeño grupal. Finalmente, se discuten los resultados y sus implicaciones teóricas así como las posibles aplicaciones prácticas.

Palabras clave: Desempeño grupal, engagement grupal, liderazgo transformacional.

\section{Metacognitive knowledge of written language: Mediation instrument and theoretical foundations}

\begin{abstract}
The current study tests the relationship between transformational leadership, team work engagement and team performance, based on the HEalthy \& Resilient Organizations (HERO) Model (Salanova, 2008; Salanova, Martinez, Cifre E Llorens, 2009) showing the mediating role of teamwork engagement in this relationship. The sample consists of 519 employees; nested in 58 work teams from 12 Small -and Medium-sized Enterprises (SMEs) from Spain. The Interclass Correlation Coefficients (ICC1 and ICC2) and the Average Deviation Index $(A D M(J))$ supports data aggregation at team level. The results, through work teams, supported the bypothesis, that is, the teamwork engagement plays a fully mediating role between transformational leadership and work team performance. Finally, theoretical and practical implications of the results are discussed.
\end{abstract}

Keywords: Team performance, teamwork engagement, transformational leadership.

Agradecimientos: Este trabajo de Investigación se enmarca en un proyecto del Ministerio de Economía y Competitividad (PSI2011-22400).

Correspondencia con las autoras: Valeria Cruz-Ortiz. Universitat Jaume I . Av. de Vicent Sos Baynat s/n. 12017 Castellón. Tlf.: +34964729580 Fax:+3496472 9262. E-mail: valeria.cruz@uji.es.

Original recibido: 31 de enero de 2012. Aceptado: 3 de mayo de 2012. 
La actual crisis económica nos lleva a diario a considerar la importancia de estrategias que promuevan el liderazgo transformacional dentro de las organizaciones, dado que los líderes son un elemento crítico dentro del contexto laboral y pueden influir en cómo las personas y equipos ven su trabajo (Christian, Garza y Slaughter, 2011). A su vez, el líder transformacional es considerado como un recurso social fundamental con que cuenta la organización para incrementar el engagement en los equipos de trabajo (y con ello su salud psicosocial), así como para incrementar los resultados organizacionales saludables a través del desempeño grupal (Salanova, 2008).

En el contexto de la Psicología Positiva, el liderazgo transformacional contribuye a fomentar organizaciones con altos niveles de bienestar psicológico y bajos niveles de estrés, malestar psicosocial y absentismo de los empleados (Llorens, Salanova y Losilla, 2009). Considerando esta función, en nuestra opinión, de acuerdo a investigaciones anteriores, pensamos que un proceso psicosocial plausible de mediar la relación entre el liderazgo transformacional y el desempeño es el engagement, dado que está comprobado que las personas que experimentan engagement, son capaces de responder a las demandas de su puesto de trabajo con eficacia y están comprometidas con sus tareas implicándose en su quehacer diario (Salanova, Martínez y Llorens, 2005). Así mismo, se comprueba que el engagement modula los efectos directos de los recursos (tal como consideramos el liderazgo transformacional) sobre el desempeño (Christian et al., 2011; Salanova y Schaufeli, 2004).

\section{Liderazgo transformacional}

Desde la propuesta del liderazgo transformacional (Bass, 1985), se considera la función de líder en el proceso motivacional de sus seguidores mediante la transformación de sus actitudes y valores así como en el aumento de su rendimiento (Molero, Cuadrado, Navas y Morales, 2007). Los líderes transformacionales son capaces de motivar a sus seguidores para que lleguen "más allá" de lo esperado (Molero, 2011). Por su parte Salanova (2008) expone que el líder transformacional es quien sabe conducir a los demás hacia un fin percibido como compartido y logra el compromiso de los equipos de trabajo, así como el de la organización.

En cuanto a la operacionalización del liderazgo transformacional, existen algunas propuestas acerca de la dimensionalidad del constructo y su evaluación. Una de las primeras y más extendida es el Multifactor Leadership Questionnaire (MLQ) diseñado por Bass y sus colaboradores (Bass, 1985; Bass y Avolio, 1990) que incluía tanto el liderazgo transaccional como el transformacional dando lugar a posteriores versiones (Molero, Recio y Cuadrado, 2010) y utilizado con muestras españolas ya sea en su versión amplia (Lopez-Zafra y Morales, 1999) como en la versión reducida (Ramos, Sarrio, Barberá y Candela, 2002). En dicho instrumento el liderazgo transformacional constaba de cuatro dimensiones: carisma, inspiración, estimulación intelectual y consideración individualizada.

Rafferty y Griffin (2004), en base a una revisión del MLQ y la investigación generada por este instrumento, proponen la redefinición de un modelo de liderazgo transformacional con cinco dimensiones: (1) visión, definida como una expresión de un cuadro idealizado del futuro basado en los valores de la organización; (2) comunicación inspiracional, son aquellos mensajes positivos sobre la organización, que construyen la motivación y la confianza; (3) estimulación intelectual, promueve el interés de los empleados a pensar el problema de nuevas maneras; (4) apoyo, se refiere a la preocupación del líder por sus empleados, teniendo en cuenta sus necesidades; y (5) reconocimiento personal, se refiere a recompensar al trabajador con alabanza y reconocimiento por el esfuerzo y logro de metas específicas. Dichos autores muestran evidencia empírica de la estructura factorial del constructo atendiendo a estas cinco dimensiones. Siguiendo esta propuesta, se ha operacionalizado el liderazgo transformacional en base a estas cinco dimensiones. 
Desempeño grupal (intra y extra rol)

En cuanto al desempeño organizacional, no existe consenso entre los autores respecto a los factores que involucra dicha variable (Salgado, 2006). Nuestra consideración del desempeño, responde a una interpretación holística que supera los aspectos estrictamente laborales. Entendemos el desempeño grupal como un valor agregado en la organización, dado por un conjunto de comportamientos de los equipos de trabajo, que contribuyen directa e indirectamente a los objetivos de la organización (Borman y Motowidlo, 1997). En este sentido, Goodman y Svyantek (1999) plantean dos dimensiones del desempeño: (1) intra rol, que se define como aquellas actividades que contribuyen directa o indirectamente a la base técnica de la organización y éstas varían entre diversos trabajos dentro de la misma organización y; (2) extra rol, que se define como aquellas actividades que no son formalmente parte del trabajo y en las que los empleados se ofrecen voluntariamente para realizarlas. En la misma, persiste un esfuerzo adicional para terminar con éxito sus actividades.

En cuanto a la relación del desempeño con el liderazgo transformacional, se ha mostrado que este aumenta la motivación de los equipos de trabajo. Puede ser directamente mediante estrategias de motivación, pero también indirectamente a través de las dimensiones que componen el liderazgo (Zaccaro, Rittman y Marks, 2001). Así el líder, a través de la influencia idealizada, motivación inspiracional y estimulación intelectual, puede favorecer el desempeño de los equipos de trabajo (Dionne, Yammarino, Atwater y Spangler, 2004). Otras veces, la investigación se ha centrado en identificar el efecto de variables mediadoras en la relación liderazgo-desempeño; como por ejemplo el efecto mediador de la potencia grupal (Schaubroeck, Lam y Cha, 2007). También la relación del líder transformacional con el desempeño extra rol, está mediada por las creencias de eficacia y engagement (Salanova, Lorente, Chambel y Martínez 2011).

\section{Engagement grupal}

Se entiende el engagement como un estado mental positivo relacionado con el trabajo y caracterizado por Vigor, Dedicación y Absorción. El Vigor, se caracteriza por altos niveles de energía y resistencia mental mientras se trabaja, a pesar de las dificultades que puedan surgir. La Dedicación, hace referencia a implicación laboral, junto con la manifestación de sentimientos de entusiasmo, inspiración, orgullo y reto por el trabajo. Se entiende por Absorción, cuando se está totalmente concentrado en el trabajo, se tiene la sensación que el tiempo "pasa volando" y hay dificultades al desconectar de lo que se está haciendo debido a las fuertes dosis de disfrute y concentración experimentadas (Schaufeli, Salanova, González-Romá y Bakker, 2002).

Estudios recientes demuestran que el engagement se relaciona positivamente con el desempeño considerado de forma global. Tanto con el desempeño intra como con el extra rol (Bakker y Bal, 2010; Schaufeli, Taris y Bakker, 2006). A su vez, Salanova, Agut y Peiró (2005) analizan la relación del engagement con el desempeño extra rol en empleados de hoteles y restaurantes, mostrando que el engagement predice el desempeño extra rol de los empleados a partir de las percepciones de los clientes. Por otro lado, un estudio reciente revela que la relación entre recursos sociales y el desempeño está mediado por el engagement (Torrente, Salanova, Llorens y Schaufeli, 2012). Sin embargo, no se encontró evidencia que relacione el liderazgo transformacional con el engagement grupal y desempeño grupal.

La Psicología Social ofrece una larga trayectoria de estudios que muestran como las creencias comunes y las experiencias afectivas surgen entre personas que trabajan juntas, que además, tienden a mostrar patrones similares de comportamiento, es decir, sienten emociones colectivas (Barsade, 2002; González-Romá, Peiró, Subirats y Mañas, 2000), así se produce un esquema mental grupal, en donde los modelos mentales del equipo reflejan las expectativas de los miembros acerca de lo que deben hacer en una situación dada y también de lo que piensan los demás miembros que se debería hacer (Klimoski y Mohammed, 1997). 
En el contexto de estas investigaciones, encuentra explicación el engagement grupal, sustentado en el "contagio emocional" que lleva a los miembros de un grupo a converger emocionalmente (Hatfield, Cacioppo y Rapson, 1994). Hay evidencia de que el desarrollo de las experiencias afectivas colectivas se forma por la influencia y persuasión social entre los miembros del grupo, o el modelado conductual (Kelly y Barsade, 2001).

En este contexto podemos pensar que, cuando los equipos de trabajo se sienten engaged, la interacción social con los compañeros de equipo conlleva comportarse y sentirse de forma similar, integrando altas dosis de vigor, dedicación por el trabajo y pasando largos períodos de absorción y concentración en lo que se está haciendo (Salanova y Schaufeli, 2009). Es por ello, que a partir de esta aseveración, en el presente trabajo, consideramos el engagement grupal e intentamos ir un paso más allá planteando que en esta relación diádica (liderazgo transformacional y desempeño) el engagement juega un papel fundamental, si lo consideramos como un proceso psicosocial emergente en los grupos (Salanova $e t$ al., 2005). Así, podemos entender el engagement grupal como un constructo motivacional, dado que, posee componentes de activación, energía, esfuerzo y persistencia y está dirigido a la consecución de objetivos.

\section{Modelo de Organizaciones Saludables y Resilientes (HERO)}

La consideración de organización saludable (DeJoy y Wilson, 2003; Sauter, Lim y Murphy, 1996) señala la importancia de atender no sólo la salud de los empleados, sino también el desarrollo de la organización como un todo, su eficacia, la supervivencia y el futuro. El Modelo de Organizaciones Saludables y Resilientes (HERO -HEalthy \& Resilient Organizations) (Salanova, 2008; Salanova et al., 2009), se refiere a como los recursos laborales se relacionan con el bienestar psicosocial de los trabajadores, favoreciendo la optimización del bienestar en el trabajo y la mejora de la calidad de vida de los trabajadores. En este modelo, las variables consideradas en el presente estudio y las relaciones establecidas entre ellas, encuentran su verdadera interpretación en la génesis de organizaciones saludables. El modelo HERO contiene tres elementos claves: (1) recursos y prácticas organizacionales saludables para estructurar y gestionar los procesos de trabajo (e.g., liderazgo transformacional) que influirían en el desarrollo del (2) capital psicológico positivo (e.g., engagement grupal) y en los (3) resultados organizacionales saludables (e.g., desempeño intra y extra rol). Estos elementos que caracterizan a una organización saludable están relacionados entre sí y se evalúan a nivel colectivo (i.e., equipos de trabajo) (Salanova, Llorens, Cifre y Martínez, 2012). Así, se propone que si la organización implementa prácticas saludables (e.g., liderazgo transformacional) éstas influirán en el desarrollo de los equipos de trabajo y en los resultados organizacionales saludables, influyendo así en las formas de estructurar y organizar los procesos de trabajo basados en una mejora constante en el tiempo (Salanova, 2008).

En este contexto, el presente estudio analiza, la relación entre el liderazgo transformacional, engagement y desempeño, mediante datos agregados a nivel de equipos de trabajo. Dado que la revisión de la literatura, señalada anteriormente, se refiere a estudios llevados a cabo con puntuaciones individuales, creemos que la perspectiva grupal adoptada en el presente trabajo, es una novedad que arrojará luz al conocimiento de los constructos colectivos.

Entendemos por equipos de trabajo aquellos trabajadores que trabajan juntos bajo el mismo supervisor y que comparten colectivamente responsabilidades sobre los resultados de su desempeño (George, 1990). Esta consideración grupal parte de las percepciones agregadas de los miembros de los equipos de trabajo. Por tal motivo, las medidas han sido obtenidas utilizando como referente los equipos de trabajo. En base a este objetivo esperamos que el engagement medie totalmente la relación entre el liderazgo transformacional y el desempeño (ver Figura 1). 
FIGURA 1

Modelo de Investigación: Modelo propuesto de mediación total

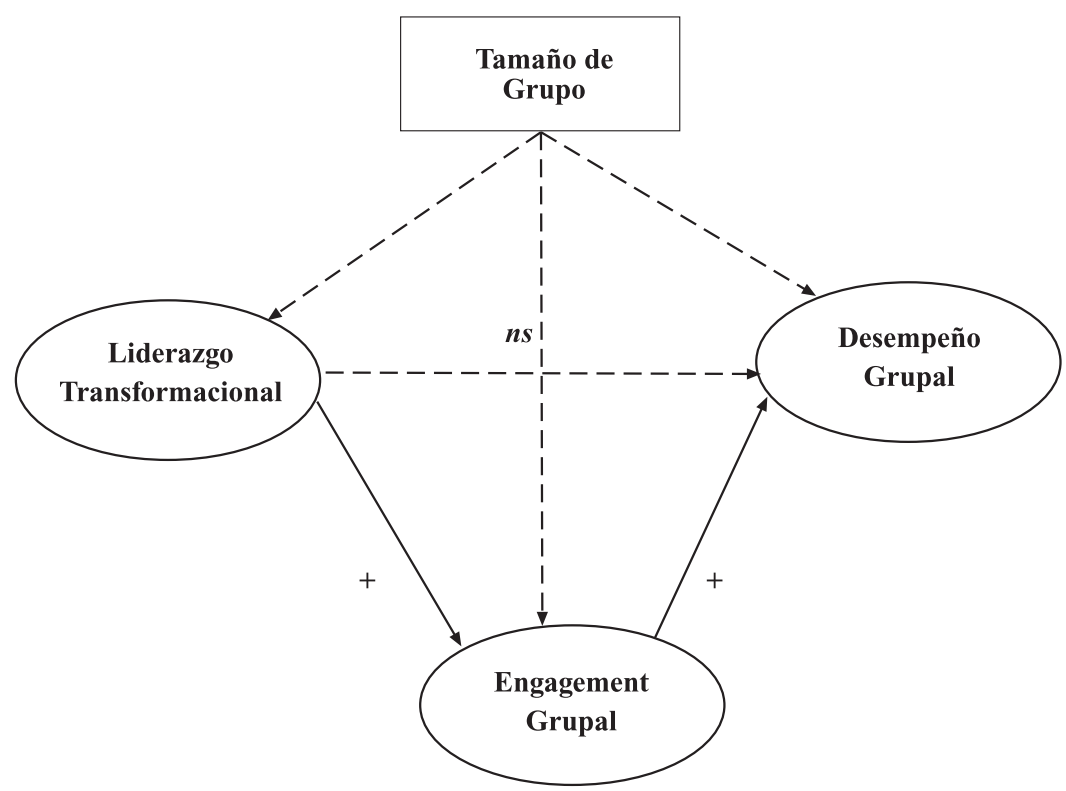

Dado el carácter colectivo de nuestro trabajo es importante señalar la consideración del tamaño del grupo como variable control. Knippenberg y Schippers (2007) muestran la importancia que la diversidad y el tamaño de los grupos tienen en el estudio de los procesos y resultados grupales. El meta-análisis de Baltes, Dickson, Sherman, Bauer y LaGanke (2002) incide en que el tamaño del grupo es un aspecto potencialmente modulador de los resultados grupales, mientras Osca y García-Salmones (2010), señalan que el tamaño afecta negativamente la satisfacción de sus miembros. Sin embargo, respecto al desempeño, los resultados de investigaciones previas no son coincidentes. Mientras Stewart y Barrick (2000) encuentran que el tamaño del grupo aumenta su eficacia, Gooding y Wagner (1985) y Osca y García-Salmones (2010) obtienen que el tamaño del grupo no tiene un efecto seguro sobre su rendimiento.

\section{Método}

\section{Muestra y Procedimiento}

La muestra está compuesta por 519 trabajadores agrupados en 58 equipos de trabajo pertenecientes a 12 Pequeñas y Medianas empresas (PyMES) españolas, de las cuales, el $84 \%$ pertenecen al sector servicios, el $8 \%$ al sector producción y el $8 \%$ al sector de industria. De la muestra el $54 \%$ son mujeres y el $70 \%$ tienen contrato indefinido. La media de antigüedad en la empresa es de 6.59 años $(D T=5.54)$. La media del tamaño de los equipos es de $8.94(D T=8.88)$ y la tasa de respuestas de los grupos es del $62 \%$.

El primer contacto con las empresas se realizó personalmente o por teléfono siendo el interlocutor, en todos los casos, un agente clave de la empresa (gerente o director de recursos humanos). Siguiendo la propuesta de McCarthy (1992), se consideraron los trabajadores con más de seis meses de antigüedad en la empresa. Cumplimentaron un cuestionario de autoinforme que consideraba como referente el equipo de trabajo al que pertenecían. La participación fue voluntaria y se garantizó la confidencialidad de las respuestas. 


\section{Medidas}

Liderazgo transformacional. Se midió a través de las cinco dimensiones del cuestionario de Rafferty y Griffin (2004) desarrollado a partir del MLQ y adaptado al castellano por Salanova et al. (2012). Para responder a los ítems, los empleados debían pensar en su jefe inmediato. Las dimensiones contenidas en dicho instrumento son las siguientes: (1) Visión $(\alpha=.74)$, compuesto por tres ítems (e.g., "Comprende perfectamente cuales son los objetivos del grupo"); (2) Comunicación inspiracional ( $\alpha=.88)$, compuesto por tres ítems (e.g., "Dice cosas positivas acerca del departamento/área"); (3).Estimulación intelectual $(\alpha=.84)$, compuesto por tres ítems (e.g., "Nos incita a pensar en viejos problemas de nuevas maneras"); (4) Apoyo $(\alpha=.93)$, compuesto por tres ítems (e.g., "Piensa en nuestras necesidades personales"); y (5) Reconocimiento ( $\alpha$ $=.96)$, compuesto por tres ítems (e.g., "Nos felicita personalmente cuando realizamos un trabajo excelente").

Engagement grupal. Se evaluó mediante la versión española (18 ítems) del cuestionario Ultrecht Work Engagement Scale (UWES; Schaufeli et al., 2002) adaptado a equipos de trabajo por Salanova, Llorens, Cifre, Martínez y Schaufeli (2003). El constructo incluye tres dimensiones: (1) Vigor $(\alpha=.92)$, compuesto por siete ítems (e.g., "Durante la realización del trabajo nos sentimos llenos de energía"); (2) Dedicación $(\alpha=.84)$, compuesto por cuatro ítems (e.g., "Disfrutamos realizando el trabajo"); y (3) Absorción ( $\alpha=.81)$, compuesto por siete ítems (e.g., "Nos dejamos llevar por el trabajo").

Desempeño grupal. Se evaluó mediante la escala de Goodman y Svyantek (1999). Para responder a los ítems los empleados debían pensar en su equipo de trabajo. La escala tiene dos dimensiones con tres ítems cada una: (1) Desempeño intra rol $(\alpha=.75)$, (e.g., "Logramos los objetivos del trabajo"); y (2) Desempeño extra rol $(\alpha=.83)$ (e.g., "Ayudamos a otros cuando estamos sobrecargados de trabajo").

Tamaño del grupo: fue considerado como variable control y medido utilizando la información disponible en las empresas estudiadas.

Todas las escalas estaban incluidas en el cuestionario HERO para organizaciones saludables y resilientes (Salanova et al., 2012). Se respondieron utilizando una escala Likert de siete puntos de anclaje que oscila de 0 (totalmente en desacuerdo/nunca) a 6 (totalmente de acuerdo/siempre). Todos los ítems tuvieron como referente el equipo de trabajo. Posteriormente se llevó a cabo la agregación de los datos a nivel grupal, considerando las puntuaciones medias de los ítems respondidos.

\section{Análisis de datos}

Tras los análisis descriptivos, se aplicó el test del factor único de Harman (Podsakoff, MacKenzie, Lee y Podsakoff, 2003) mediante el programa AMOS 18.0 (Arbuckle, 1997) para poner a prueba la estructura factorial de las dimensiones de las variables del estudio (liderazgo transformacional, engagement y desempeño), con el fin de verificar si hubo sesgo de la varianza del método común. En segundo lugar, se utilizaron dos enfoques complementarios de índices de consistencia y acuerdo para cada una de las escalas utilizadas (Bliese, 2000): El primero de ellos fue el Coeficiente de Correlación Intraclase: CCI $_{1}$ (referido a la variabilidad individual explicada por la pertenencia al grupo) y $\mathrm{CCI}_{2}$ (que considera la fiabilidad de las medidas grupales), ejecutado a partir del programa PASW 18.0. El criterio para el $\mathrm{CCI}_{1}$ y el $\mathrm{CCI}_{2}$ es de .12 y .60 o superior, respectivamente (Bliese, 2000). Desde otro enfoque, mas centrado en el acuerdo intragrupo, se ha considerado el Índice de Desviación Promedio $\left(\mathrm{AD}_{\mathrm{M}(\mathrm{J})}\right)$ (Burke y Dunlap, 2002). Además, se realizaron Análisis de Varianza (ANOVA) con el objetivo de evaluar la variabilidad entre los equipos de trabajo en las escalas evaluadas. En tercer lugar, realizamos intercorrelaciones entre las variables agregadas a nivel de equipos de trabajo. Finalmente, se utilizó el programa estadístico AMOS 18.0 para ejecutar Modelos de Ecuaciones Estructurales (SEM). 
El método de estimación utilizado fue el de máxima verosimilitud. Se evaluaron dos índices absolutos para evidenciar la bondad del ajuste de los modelos: (1) el estadístico Chi- cuadrado $\left(\chi^{2}\right)$; y (2) el Root Mean Square Error of Approximation (RMSEA). El $\chi^{2}$ es sensible al tamaño de la muestra por lo que se recomienda el uso de índices relativos para evaluar la bondad del ajuste de los modelos (Bentler, 1990; Marsh, Balla y Hau, 1996). Por tanto, fueron evaluados cuatro índices relativos de bondad del ajuste: (1) Comparative Fit Index (CFI); (2) Normed Fit Index (NFI); (3) Tucker-Lewis Index (TLI); y (4) Incremental Fit Index (IFI). Para el RMSEA valores menores que .05 se consideran como un ajuste excelente, .08 es considerado como un ajuste aceptable, y valores superiores a .10 indican una explicación inadecuada de los datos (Browne y Cudeck, 1993). En los demás casos, CFI, NFI, TLI e IFI al tratarse de índices de ajuste relativos, un valor igual o mayor de .90, se considera un índice de buen ajuste (Hu y Bentler, 1999).

\section{Resultados}

\section{Análisis descriptivos y agregación de datos}

Los resultados de los análisis descriptivos se muestran en la tabla I. Los índices de asimetría (entre -.22 y .60) y curtosis (entre -.72 y .80) mostraron valores óptimos (George y Mallery, 2001) así como también valores aceptables de fiabilidad de las escalas (Nunnaly y Bernstein, 1994). En el test del factor único de Harman (Podsakoff et al., 2003), los resultados revelaron unos índices de ajuste pobre $\chi^{2}(35)=$ $248.76, p<.000, \mathrm{RMSEA}=.33, \mathrm{CFI}=.55, \mathrm{NFI}=.52, \mathrm{TLI}=42, \mathrm{IFI}=.56$. Adicionalmente se comparó este modelo de factor único, con otro de tres factores, el cual reveló índices de ajuste aceptables $\chi^{2}(32)=54.65, p<.008$, RMSEA $=.11, \mathrm{CFI}=$ $.95, \mathrm{NFI}=.90, \mathrm{TLI}=.94, \mathrm{IFI}=.95$, apoyando la existencia de independencia de las tres variables consideradas. Por tanto, podemos considerar que el sesgo de la varianza común no es una deficiencia seria en esta base de datos.

En cuanto a los análisis sobre consistencia y acuerdo, la tabla I muestra los índices de acuerdo de todas las variables incluidas en el estudio agregadas a nivel de equipos de trabajo. El rango para el $\mathrm{CCI}_{1}$ (entre .15 y .30), $\mathrm{CCI}_{2}$ (entre.60 y .80) es satisfactorio (Bliese, $2000)$ y el $\mathrm{AD}_{\mathrm{M}(\mathrm{J})}$, (entre .54 y .10) también cumple con el criterio promedio recomendado (Burke y Dunlap, 2002). También los análisis de varianza (ANOVA) muestran evidencia de la variabilidad entre grupos. De estos resultados se puede concluir la posibilidad de agregar las percepciones de los miembros a nivel de equipo. La base de datos agregada se construyó con las puntuaciones medias grupales. Agregados los datos (Tabla I), se comprobó la correlación positiva y significativa entre las dimensiones de los constructos (entre .21 y $.80 ; p<.001)$.

\section{Ajuste del modelo}

Para responder al objetivo del presente trabajo se llevaron a cabo análisis de Modelos de Ecuaciones Estructurales (SEM). Las escalas agregadas a nivel de equipos de trabajo se consideraron como variables latentes con sus correspondientes dimensiones (liderazgo transformacional, cinco indicadores: visión, comunicación inspiracional, estimulación intelectual, apoyo y reconocimiento; engagement, tres indicadores: vigor, dedicación y absorción; desempeño, dos indicadores: intra y extra rol). En este análisis se consideró el tamaño del grupo como variable control.

Se experimentó con dos Modelos de Ecuaciones Estructurales (SEM). El M1, donde la relación entre liderazgo transformacional y desempeño está totalmente mediada por el engagement. El M2 donde esta mediación es parcial, considerando también los efectos directos del liderazgo transformacional sobre el desempeño. Los resultados, ponen de manifiesto el buen ajuste de los modelos y que todos los índices 
8 Revista de Psicología Social, 2013, 28 (2), pp. 0-0

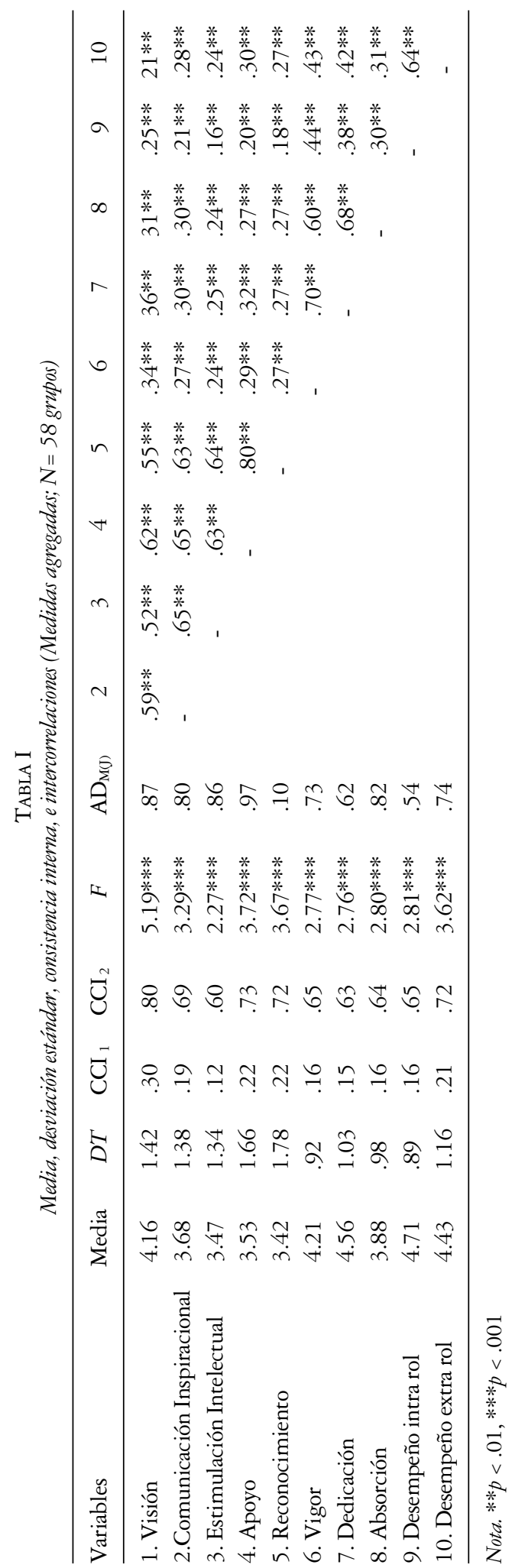


cumplen los criterios. El test de Chi-cuadrado entre el M1 (Modelo de Mediación Total) y el M2 (Modelo de Mediación Parcial), muestra que entre ambos modelos no hay diferencias significativas Delta $\chi^{2}(1)=.003$, ns. Dado que el M1 es más parsimonioso, optamos por este modelo que propone la mediación total del engagement entre el liderazgo transformacional y desempeño. Es importante remarcar, que el liderazgo transformacional explica un $27 \%$ de la varianza en el engagement, que a su vez explica el $55 \%$ de la varianza del desempeño. Además se llevaron a cabo análisis adicionales donde se puso a prueba el modelo parcialmente mediado (M2), con un tercer modelo (M3), de mediación total que constriñe la relación entre engagement y desempeño mediante el coeficiente no estandarizado de esta relación en el M1 (Peiró, González- Romá, Ripoll y Gracia, 2001; Salanova et al., 2005). Los resultados del M3 cumplen todos los criterios de ajuste para los índices de bondad, la diferencia de chicuadrado entre el M2 y el M3 no fue significativa. De lo cual se desprende evidencia de la mediación total que el engagement ejerce en la relación liderazgo transformacional con desempeño (ver Tabla II).

TABLA II

Índices del Modelo de Ecuaciones Estructurales ( $N=58$ grupos)

\begin{tabular}{lccccccccc}
\hline Modelos & $\chi^{2}$ & $d f$ & CFI & NFI & TLI & IFI & RMSEA & $\Delta \chi^{2}$ & $\Delta d f$ \\
\hline M1 & 52.503 & 39 & .97 & .90 & .96 & .97 & .078 & & \\
M2 & 52.500 & 38 & .97 & .92 & .95 & .97 & .082 & & \\
M3 & 54.648 & 32 & .95 & .89 & .93 & .95 & .111 & & \\
M2-M1 & & & & & & & & $0.003 n s$ & 1 \\
M3-M2 & & & & & & & & $-2.15 \mathrm{~ns}$ & 6 \\
\hline
\end{tabular}

Nota $. \chi^{2}=$ Chi-square $d f=$ degrees of freedom; RMSEA = Root Mean Square Error of Approximation; CFI = Comparative Fit Index ; NFI = Normed Fit Index; TLI = Tucker-Lewis Index; IFI = Incremental Fit Index; $n s=$ no significativo

Para abordar el análisis de la mediación, se siguió a Baron y Kenny (1986) para variables latentes: (1) el liderazgo transformacional se relaciona positiva y significativamente con el desempeño $(\beta=.25, p=.01)$; (2) el liderazgo transformacional se relaciona positiva y significativamente con el engagement $(\beta=.34, p=.001)$; (3) el liderazgo transformacional se relaciona positivamente con el engagement y éste con el desempeño ( $\beta=.79, p=.001)$; y (4) cuando se considera el efecto directo del liderazgo transformacional sobre el desempeño, la relación no es significativa $(\beta=.01, p$ $=.85)$. la relación entre el liderazgo transformacional con el engagement y éste con el desempeño considerando también el efecto directo del liderazgo transformacional sobre el desempeño. Esta última relación no es significativa $(\beta=.01, p=.85)$. A la vista de estos resultados, se considera que el engagement media totalmente la relación entre el liderazgo transformacional y el desempeño.

Además, se han ejecutado las pruebas de Sobel (1982) con el fin confirmar el efecto indirecto del liderazgo transformacional sobre el desempeño a través del engagement. Los resultados (Sobel test $=2.48, p<.01$ ), apoyan dicha relación y por lo tanto que hay mediación total.

Por otro lado, considerando la variable tamaño del grupo se muestra que esta variable control, tiene una influencia negativa y significativa en la variable del liderazgo transformacional, pero no tiene efecto en las variables del engagement y desempeño.

En resumen, los resultados evidencian que el engagement media de forma total entre el liderazgo transformacional y el desempeño con lo que se cumple el objetivo y confirma la hipótesis del presente trabajo y que se muestra gráficamente en la figura 2. 


\section{Revista de Psicología Social, 2013, 28 (2), pp. 0-0}

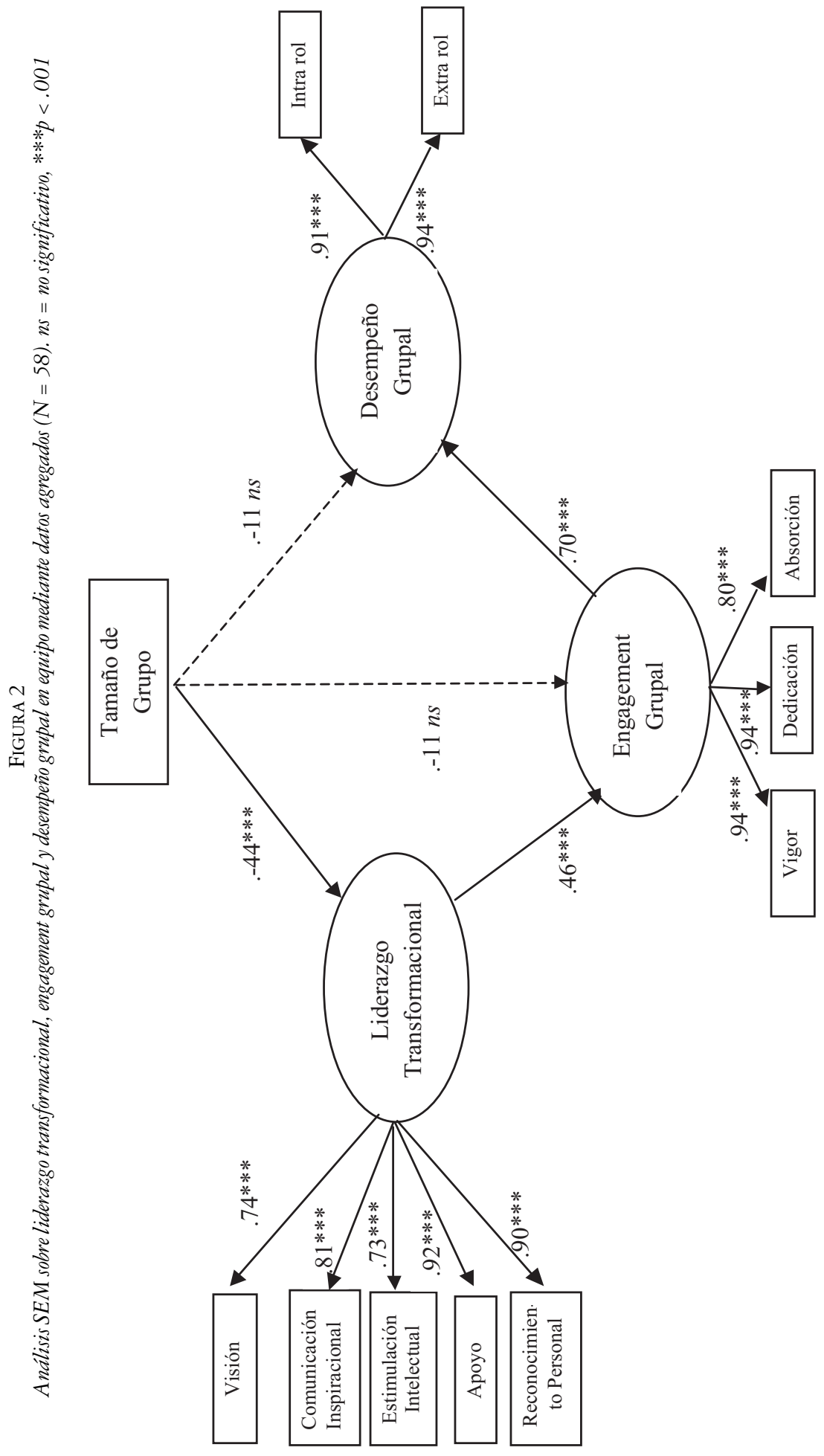




\section{Discusión}

El objetivo de esta investigación era analizar la relación que el liderazgo transformacional tiene con el desempeño y el papel que el engagement tiene en esta relación. Para ello se ha puesto a prueba la mediación total y parcial del engagement en la relación entre el liderazgo transformacional y desempeño, en 58 unidades de equipos de trabajo de diferentes PyMEs. A través de los análisis realizados, probamos el papel mediador del engagement (vigor, dedicación y absorción) entre el liderazgo transformacional y el desempeño (intra y extra rol), considerando la agregación de las percepciones de los miembros a nivel de equipos de trabajo. Con ello, nuestra investigación hace un aporte innovador, dado que la relación de las variables de esta investigación ha sido considerada a nivel de equipos de trabajo. Además se consideró el tamaño del grupo como variable control, dado la variabilidad en tamaño existente. Los resultados muestran que el tamaño de los equipos de trabajo influye en las puntuaciones que los miembros de los equipos asignaron a su supervisor. Esto imprime importancia al tamaño del grupo, ya que a menor número de miembros en los equipos de trabajo, mayor será el efecto del líder transformacional sobre el equipo que supervisa.

Como se expuso al inicio de nuestro trabajo, pocas investigaciones analizaron los procesos psicosociales subyacentes que pueden estar influyendo en la relación entre el liderazgo transformacional y el desempeño. Por eso nuestros resultados se han dirigido a estudiar los efectos mediacionales en esta relación, evidenciando que el efecto del liderazgo transformacional sobre el desempeño, no es directo, sino mediado por el engagement. Esto supone que los esfuerzos del líder y los postulados del liderazgo transformacional se ven mediados por el efecto del engagement. Los equipos de trabajo que están engaged, están comprometidos con sus tareas, y no sólo centran su esfuerzo en cumplimentar los objetivos propuestos por las empresas, sino que también están emocionalmente conectados a sus tareas. Tal como se ha mostrado en la revisión bibliográfica (Bakker y Bal, 2010; Schaufeli et al., 2006), el engagement se relaciona con el desempeño, tanto intra como extra rol, pero además, aquí se ha mostrado que esta relación existe también a nivel colectivo cuando se considera el engagement grupal. Los equipos de trabajo experimentan emociones en la ejecución de las tareas, que mejoran su desempeño grupal a través de una mayor conexión entre los compañeros de trabajo en pos de lograr los objetivos propuestos por la organización.

En suma, los resultados apoyan la hipótesis planteada en esta investigación y podemos indicar que el objetivo de este estudio se ha cumplido. Esta consideración es importante ya que pone de manifiesto el valor del bienestar psicosocial del grupo y en concreto el engagement, para aprovechar los recursos sociales que posee la organización (liderazgo transformacional). Sin embargo, la disponibilidad de recursos sociales como es el liderazgo transformacional, no garantiza el desempeño del grupo ya que esta relación se puede ver afectada por el engagement.

\section{Implicaciones Teóricas y Prácticas}

El presente estudio muestra diferentes implicaciones teóricas y prácticas. A nivel teórico, amplía la definición de engagement individual a engagement grupal y contribuye al conocimiento del engagement como estado afectivo, cognitivo y conductual que media la relación entre el liderazgo transformacional y desempeño. Esta relación positiva ofrece evidencia al Modelo HERO (Salanova, 2008; Salanova et al., 2009; Salanova et al., 2012) ya que analiza la interacción propuesta por el modelo entre sus tres elementos claves: recursos y prácticas organizacionales saludables (e.g., liderazgo transformacional), empleados saludables (e.g., engagement) y resultados saludables (e.g., desempeño) utilizando niveles superiores de análisis (i.e., equipos de trabajo). Además, los resultados contribuyen a la investigación mostrando los beneficios de potenciar los aspectos positivos en contextos laborales, en nuestro caso el engagement, por su importante papel en los resultados grupales. 
Desde el punto de vista de la práctica organizacional, estos resultados son muy interesantes ya que en ocasiones la inversión que se lleva a cabo en recursos sociales no tiene los efectos deseados sobre el desempeño grupal debido a que no se tienen en cuenta los procesos mediadores en esta relación. Por ello, esta investigación interesa a profesionales de recursos humanos de cara a fomentar la función del líder transformacional dentro de sus equipos de trabajo, desde la formación de líderes transformacionales cuyo objetivo sea promocionar la salud psicosocial dentro de la organización. Invertir en la formación de líderes transformacionales posibilitaría incrementar el engagement dentro de los equipos de trabajo y sobre todo el desempeño grupal, lo cual la organización debería interpretar como un paso importante en la producción, pero sobre todo, en los resultados saludables que se obtendrían.

Podemos concluir que las organizaciones deben fortalecer el engagement en los equipos de trabajo, esto tendrá una relación positiva entre el liderazgo transformacional y desempeño.

\section{Limitaciones e investigaciones futuras}

Una de las posibles limitaciones de este estudio, es que los datos fueron obtenidos a través de medidas de autoinforme. A pesar de ello, los datos no se trataron a nivel individual sino que se consideraron percepciones agregadas de equipos de trabajo para el liderazgo transformacional, engagement y desempeño. Como consecuencia, al utilizar estos datos agregados a nivel de equipos de trabajo podemos aumentar la validez de las puntuaciones, teniendo en cuenta que tratamos con intersubjetividades compartidas entre los miembros de los equipos, y no subjetividades individuales. Otra limitación es que utilizamos una muestra de conveniencia. A pesar de ello, la muestra utilizada incluye diferentes equipos de trabajo pertenecientes a diversas empresas que a su vez pertenecen a diferentes sectores económicos. Futuros estudios deben estar orientados a desentrañar los caminos causales por medio de técnicas longitudinales. El uso de metodología multinivel es recomendado para explorar estudios longitudinales en donde se relaciona el nivel de la organización y variables de niveles más bajos, como lo son los recursos humanos en donde sería interesante explorar las diferencias de las percepciones entre supervisores y empleados respecto al liderazgo transformacional, relacionándolas con otras variables, como por ejemplo, el capital psicológico positivo (engagement) y desempeño.

\section{Nota final}

Este estudio ha permitido hacer explícita la relación entre liderazgo transformacional, engagement y desempeño utilizando datos agregados. Esto supone una contribución al desarrollo de la Psicología Ocupacional Positiva al poner de manifiesto la importancia de los aspectos positivos en el trabajo. Por otro lado aporta datos interesantes para el quehacer diario de los profesionales de los recursos humanos en su afán por desarrollar Organizaciones Saludables y Resilientes.

\section{Referencias}

ArbuCKLe, J. L. (1997). Amos users' guide versión 4.0. Chicago, IL: Smallwaters Corporation.

BAKKer, A. N. \& BAL, P. M. (2010). Weekly work engagement and performance: A study among starting teachers. Journal of Occupational and Organizational Psychology, 83, 189-206.

Baltes, B. B., Dickson, M. W., Sherman, M. P., Bauer, C. C. \& LaGanke, J. S. (2002). Computermediated communication and group decision making: A meta-analysis. Organizational Behavior and Human Decision Processes, 87, 156-179.

BARON, R. M. \& KENNY, D. A. (1986). The moderator- mediator variable distinction in social psychological research: Conceptual, strategic and statistical consideration. Journal of Personality and Social Psychology, 51, 1173-1182.

BARSADE, S. G. (2002). The ripple effect: Emotional contagion and its influence on group behavior. Administrative Science Quarterly, 47, 644-675. 
BAss, B. M. (1985). Leadership and performance beyond expectations. Nueva York: The Free Press.

Bass, B. M. \& Avolio, B. J. (1990). Transformational leadership development: Manual for the Multifactor Leadership Questionnaire. Palo Alto, CA: Consulting Psychologists Press Inc.

Bentler, P. M. (1990). Comparative fit indexes in structural equation models. Psychological Bulletin, 107, 238-246.

Bliese, P. D. (2000). Within-group agreement, non-independence, and reliability. Implications for data aggregation and analysis. En K. J. Klein, \& S. W. J. Kozlowski (Eds.), Multilevel theory, research, and, methods in organization (pp. 349-381). San Francisco: Jossey-Bass.

BORMAN, W. C. \& MOTOWIDLO, S. (1997). Task performance and contextual performance: The meaning for personnel selection research. Human Performance, 10, 99-109.

Browne, M. W. \& CudeCK, R. (1993). Alternative ways of assessing model fit. En K. A. Bollen \& J. S. Long (Eds.), Testing structural equation models (pp. 136-162). Newbury Park, CA: Sage.

BurKe, M. J. \& DunLAP, W. P. (2002). Estimating interrater agreement with the average deviation index: A user's guide. Organizational Research Methods, 5, 159-172.

Christian, M. S., Garza, A. S. \& Slaughter, J. E. (2011). Work engagement: A quantitative review and test of its relations with task and contextual performance. Personnel Psychology, 64, 89-136.

DeJoy, D. M. \& Wilson, M. G. (2003). Organizational health promotion: Broadening the horizon of workplace health promotion. American Journal of Health Promotion, 17, 337-341.

Dionne, S. D., Yammarino, F. J., Atwater, L. E. \& Spangler, W. D. (2004). Transformational leadership and team performance. Journal of Organizational Change Management, 17, 177-193.

George, J. M. (1990). Personality, affect, and behaviors in groups. Journal of Applied Psychology, 75, 107-116.

GeOrge, D. \& MALLERY, M. (2001). Using SPSS for Windows step by step: A simple guide and reference. Boston, MA: Allyn and Bacon.

GonzÁlez-Romá, V., Peiró, J. M., Subirats, M. \& Mañas, M. A. (2000). The validity of affective work team climates. En M. Vartiainen, F. Avallone, \& N. Anderson (Eds.), Innovative theories, tools and practices in work and organizational psychology (pp. 97-109). Götting: Hogrefe \& Huber.

Gooding, R. Z. \& WagneR, J. A. (1985). A meta-analytic review of the relationship between size and performance: The productivity and efficiency of organizations and their subunits. Administrative Science Quarterly, 30, 462-481.

Goodman, S. A. \& Svyantek, D. J. (1999). Person-organization fit and contextual performance: Do shared values matter. Journal of Vocational Behavior, 55, 254-275.

HatField, E., CACIOPPO, J. \& RAPSON, R. L. (1994). Emotional contagion. Nueva York: Cambridge University Press.

Hu, L. \& Bentler, P. M. (1999). Cutoff criteria for fit indices in covariance structure analysis: Conventional criteria versus new alternatives. Structural Equation Modeling, 6, 1-55.

Kelly, J. \& BARsade, S. G. (2001). Mood and emotions in small groups and work teams. Organizational Behavior and Human Decision Processes, 86, 99-130.

KLIMOSKI, R. \& MOHAMmED, S. (1997). Team mental model: Construct or metaphor? Journal of Management, 20, 403-437.

KNipPEnBerg, D. \& SCHIPPers, M. C. (2007). Work group diversity. Annual Review of Psychology, 58, 515541.

Llorens, S., Salanova, M. \& Losilla, J. (2009). Liderazgo transformacional y capital psicológico positivo: un estudio de caso en una empresa de construcción. Directivos Construcción, 220, 48-56.

LÓPEZ-Zafra, E. \& Morales, F. (1999). Niveles de análisis en el estudio del liderazgo carismático. Revista de Psicología Social, 14, 181-197.

Marsh, H. W., Balla, J. R. \& HaU, K. T. (1996). An evaluation of incremental fit indices: A clarification of mathematical and empirical properties. En G. A. Marcoulides, \& R. E.Schumacker (Eds.), Advanced structural equation modeling, issues and techniques (pp. 315-353). Mahwah, NJ: Lawrence Erlbaum Associates Publishers.

MCCARTHY, J. P. (1992). Focus from the start - employee orientation programs - Orientation. HR Magazine, 37, 77-83.

Molero, F. (2011). Liderazgo transformacional y carismático. En F. Molero \& J. F. Morales (Eds.), Liderazgo becho y ficción. Visiones actuales (pp. 117-143). Madrid: Alianza.

Molero, F., Cuadrado, I., Navas, M. \& Morales, F. (2007). Relations and effects of transformational leadership: A comparative analysis with traditional leaership styles. The Spanish Journal of Psychology, 10, 358-368.

Molero, F., Recio, P. \& CuAdrado, I. (2010). Liderazgo transformacional y liderazgo transaccional: un análisis de la estructura factorial del Multifactor Leadership Questionnaire (MLQ) en una muestra española. Psicothema, 22, 495-501.

Nunnaly, J. C. \& Bernstein, I. H. (1994). Psychometric theory. Nueva York: McGraw-Hill.

Osca, A. \& García-Salmones, L. (2010). El impacto del tamaño y la diversidad en los procesos y resultados grupales. Psicothema, 22, 137-142.

Peiró, J. M., González-Romá, V., Ripoll, P. \& Gracia, F. J. (2001). Role stress and work team variables in primary health care teams: A structural equations model. En J. De Jonge, P. Vlerick, A. Büssing, \& 


\section{Revista de Psicología Social, 2013, 28 (2), pp. 0-0}

W. B. Schaufeli (Eds.), Organizational psychology and health care at the start of the new millennium (pp. 105122). München: Rainer Hampp Verlag.

Podsakoff, P. M., MacKenzie, S. M., Lee, J. \& Podsakoff, N. P. (2003). Common method variance in behavioral research: A critical review of the literature and recommended remedies. Journal Applied of Psychology, 88, 879-903.

Ramos, A., Sarrió, M., Barberá, E. \& Candela, C. (2002). Mujeres directivas y demandas organizacionales. Revista de Psicología Social, 17, 183-192

RAfFerty, A. E. \& Griffin. M. A. (2004). Dimensions of transformational leadership: Conceptual and empirical extensions. The Leadership Quarterly, 15, 329-354.

SAlANOVA, M. (2008). Organizaciones saludables y desarrollo de recursos humanos. Estudios Financieros, 303, $179-214$

Salanova, M., Agut, S. \& Peiró, J. M. (2005). Linking organizational resources and work engagement to employee performance and customer loyalty: The mediation of service climate. Journal of Applied Psychology, 90, 1217-1227.

Salanova, M., Llorens, S., Cifre, E. \& Martínez, I. M. (2012). Development and validation of a Healthy and Resilient Organizations (HERO) model. Manuscrito sometido para su publicación.

Salanova, M., Llorens, S., Cifre, E., Martínez, I. M. \& Schaufeli, W. B. (2003). Perceived collective efficacy, subjective well-being and task performance among electronic groups: An experimental study. Small Groups Research, 34, 43-73.

Salanova, M., Lorente, L., Chambel, M. J. \& MartíneZ, I. M. (2011). Linking transformational leadership to extra rol behavior: The mediating role of self-efficacy and work engagement. Journal of Advanced Nursing, 67, 2256-2266.

Salanova, M., Martínez, I. M., Cifre, E. \& Llorens, S. (2009). La salud ocupacional desde la perspectiva psicosocial: aspectos teóricos y conceptuales. En M. Salanova (Ed.), Psicología de la salud ocupacional (pp. 2761). Madrid: Síntesis.

Salanova, M., Martínez, I. M. \& Llorens, S. (2005). ¿Cómo se relacionan los obstáculos y facilitadores organizacionales con el burnout docente? Revista de Psicología del Trabajo y de las Organizaciones, 21, 37-54.

SAlanOVA, M. \& SCHAUfELI, W. B. (2004). Engagement: un reto emergente para la dirección de los recursos humanos. Revista de Trabajo y Seguridad Social, 261, 109-138.

Salanova, M. \& SCHAUfeli, W. B. (2009). El engagement en el trabajo. Cuando el trabajo se convierte en pasión. Madrid. Editorial Alianza.

SAlgado, E. (2006). Dos textos: sobre el desempeño individual en las organizaciones. Monografias de Administración, 93, 1-54.

SAuter, S. L., Lim, S. \& MurPhy, L. R. (1996). Organizational health: A new paradigm for occupational stress research at NIOSH. Occupational Mental Health, 4, 248-254.

SchaubroeCK, J., LAM, S. \& CHA, S. A. (2007). Embracing transformational leadership: Team values and the impact of leader behavior on team performance. Journal of Applied Psychology, 92, 1020-1030.

Schaufeli, W. B., Salanova, M., González- Romá, V. \& Bakker, A. B. (2002). The measurement of engagement and burnout: A confirmative analytic approach. Journal of Happiness Studies, 3, 71-92.

Schaufeli, W. B., Taris, T. W. \& Bakker, A. B. (2006). Dr. Jeckyll or Mr. Hyde: On the differences between work engagement and workaholism. En R. J. Burke (Eds.), Research companion to working time and work addiction (pp. 193-217). Northhampton, UK: Edward Elgar.

SoBEL, M. E. (1982). Asymptotic confidence intervals for indirect effects in structural equation models. En S. Leinhardt (Eds.), Sociological methodology (pp. 290-312). Washington, DC: American Sociological Association.

Stewart, G. L. \& BARrick, M. R. (2000). Team structure and performance: Assessing the mediating role of intrateam process and the moderating role of task type. Academy of Management Journal, 43, 135-148.

Torrente, P., Salanova, M., Llorens, S. \& Schaufeli, W. B. (2012). Teams make it work: How team work engagement mediates between social resources and performance in teams. Psicothema, 24, 106-112.

Zaccaro, S. J., Rittman, A. L. \& MARKs, M. A. (2001). Team Leadership. The Leadership Quarterly 12 , 451483. 